\title{
Poincaré Problem for Nonlinear Elliptic Equations of Second Order in Unbounded Domains
}

\author{
Guochun Wen \\ LMAM, School of Mathematical Sciences, Peking University, Beijing, China \\ Email: wengc@math.pku.edu.cn
}

Received September 27, 2012; revised November 2, 2012; accepted November 10, 2012

\begin{abstract}
In [1], I. N. Vekua propose the Poincaré problem for some second order elliptic equations, but it can not be solved. In [2], the authors discussed the boundary value problem for nonlinear elliptic equations of second order in some bounded domains. In this article, the Poincaré boundary value problem for general nonlinear elliptic equations of second order in unbounded multiply connected domains have been completely investigated. We first provide the formulation of the above boundary value problem and corresponding modified well posed-ness. Next we obtain the representation theorem and a priori estimates of solutions for the modified problem. Finally by the above estimates of solutions and the Schauder fixed-point theorem, the solvability results of the above Poincaré problem for the nonlinear elliptic equations of second order can be obtained. The above problem possesses many applications in mechanics and physics and so on.
\end{abstract}

Keywords: Poincaré Boundary Value Problem; Nonlinear Elliptic Equations; Unbounded Domains

\section{Formulation of the Poincaré Boundary Value Problem}

Let $D$ be an $(N+1)$-connected domain including the infinite point with the boundary $\Gamma=\bigcup_{j=0}^{N} \Gamma_{j}$ in $\mathbb{C}$, where $\Gamma \in C_{\mu}^{2}(0<\mu<1)$. Without loss of generality, we assume that $D$ is a circular domain in $|z|>1$, where the boundary consists of $N+1$ circles $\Gamma_{0}=\Gamma_{N+1}=\{|z|=1\}$, $\Gamma_{j}=\left\{\left|z-z_{j}\right|=r_{j}\right\}, j=1, \cdots, N$ and $z=\infty \in D$. In this article, the notations are as the same in References [1-8]. We consider the second order equation in the complex form

$$
\left\{\begin{array}{l}
u_{z \bar{z}}=F\left(z, u, u_{z}, u_{z z}\right)+G\left(z, u, u_{z}\right), \\
F=\operatorname{Re}\left[Q u_{z z}+A_{1} u_{z}\right]+\varepsilon A_{2} u+A_{3}, \\
G=G\left(z, u, u_{z}\right), Q=Q\left(z, u, u_{z}, u_{z z}\right), \\
A_{j}=A_{j}\left(z, u, u_{z}\right), j=1,2,3,
\end{array}\right.
$$

satisfying the following conditions.

Condition C. 1) $Q(z, u, w, U), A_{j}(z, u, w)(j=1,2,3)$ are continuous in $u \in \mathbb{R}, w \in \mathbb{C}$ for almost every point $z \in D, U \in \mathbb{C}$, and $Q=0, A_{j}=0(j=1,2,3)$ for $z \notin D$.

2) The above functions are measurable in $z \in D$ for all continuous functions $u(z), w(z)$ in $\bar{D}$, and satisfy

$$
\begin{aligned}
& L_{p, 2}\left[A_{j}(z, u, w), \bar{D}\right] \leq k_{0}, j=1,2, \\
& L_{p, 2}\left[A_{3}(z, u, w), \bar{D}\right] \leq k_{1},
\end{aligned}
$$

in which $p_{0}, p\left(2<p_{0} \leq p\right), k_{0}, k_{1}$ are non-negative constants.

3) The Equation (1.1) satisfies the uniform ellipticity condition, namely for any number $u \in \mathbb{R}$ and $w, U_{1}$, $U_{2} \in \mathbb{C}$, the inequality

$$
\left|F\left(z, u, w, U_{1}\right)-F\left(z, u, w, U_{2}\right)\right| \leq q_{0}\left|U_{1}-U_{2}\right|,
$$

for almost every point $z \in D$ holds, where $q_{0}(<1)$ is a non-negative constant.

4) For any function $u(z) \in C(\bar{D}), w(z) \in W_{p, 2}^{1}(D)$, $G(z, u, w)$ satisfies the condition

$$
G\left(z, u, u_{z}\right)=B_{1}\left|u_{z}\right|^{\sigma}+B_{2}|u|^{\tau}, 0<\sigma, \tau<\infty,
$$

in which $B_{j}=B_{j}\left(z, u, u_{z}\right)(j=1,2)$ satisfy the condition

$$
L_{p, 2}\left[B_{j}, \bar{D}\right] \leq k_{0}<\infty, j=1,2,
$$

with a non-negative constant $k_{0}$.

Now, we formulate the Poincare boundary value problem as follows.

Problem $P$. In the domain $D$, find a solution $u(z)$ of Equation (1.1), which is continuously differentiable in $\bar{D}$, and satisfies the boundary condition

$$
\begin{aligned}
& \frac{1}{2} \frac{\partial u}{\partial v}+\varepsilon c_{1}(z) u=c_{2}(z), \\
& \text { i.e. } \operatorname{Re}\left[\overline{\lambda(z)} u_{z}\right]+\varepsilon c_{1}(z) u=c_{2}(z), z \in \Gamma,
\end{aligned}
$$

in which $v$ is any unit vector at every point on $\Gamma=\partial D$, $\lambda(z)=\cos (v, x)-i \cos (v, y), \quad c_{1}(z)$ and $c_{2}(z)$ are 
known functions satisfying the conditions

$$
C_{\alpha}[\lambda, \Gamma] \leq k_{0}, C_{\alpha}\left[c_{1}, \Gamma\right] \leq k_{0}, C_{\alpha}\left[c_{2}, \Gamma\right] \leq k_{2},
$$

where $\varepsilon(>0), \alpha(1 / 2<\alpha<1), k_{0}, k_{2}$ are non-negative constants.

If $\cos (v, n)=0$ and $c_{1}=0$ on $\Gamma$, where $n$ is the outward normal vector on $\Gamma$, then Problem $P$ is the Dirichlet boundary value problem (Problem $D$ ). If $\cos (v, n)=1$ and $a_{1}=0$ on $\Gamma$, then Problem $P$ is the Neumann boundary value problem (Problem $N$ ), and if $\cos (v, n)>0$, and $c_{1} \geq 0$ on $\Gamma$, then Problem $P$ is the regular oblique derivative problem, i.e. the third boundary value problem (Problem III or $O$ ). Now the directional derivative may be arbitrary, hence the boundary condition is very general.

The integer

$$
K=\frac{1}{2 \pi} \Delta_{\Gamma} \arg \lambda(z)
$$

is called the index of Problem $P$. When the index $K<0$, Problem $P$ may not be solvable, and when $K \geq 0$, the solution of Problem $P$ is not necessarily unique. Hence we consider the well-posedness of Problem $P$ with modified boundary conditions.

Problem Q. Find a continuous solution $[w(z), u(z)]$ of the complex equation

$$
\left\{\begin{array}{l}
w_{\bar{z}}=F\left(z, u, w, w_{z}\right)+G(z, u, w), \\
F=\operatorname{Re}\left[Q w_{z}+A_{1} w\right]+\varepsilon A_{2} u+A_{3}, G=B_{1}|w|^{\sigma}+B_{2}|u|^{\tau},
\end{array}\right.
$$

satisfying the boundary condition

$$
\operatorname{Re}[\overline{\lambda(z)} w(z)]+\varepsilon c_{1}(z) u=c_{2}(z)+h(z), z \in \Gamma,
$$

and the relation

$$
u(z)=-2 \operatorname{Re} \int_{1}^{z}\left[\frac{w(z)}{z^{2}}-\sum_{j=1}^{N} \frac{i d_{j} z_{j}}{z\left(z-z_{j}\right)} \mathrm{d} z\right]+b_{0},
$$

where $d_{j}(j=1, \cdots, N)$ are appropriate real constants such that the function determined by the integral in (1.8) is single-valued in $D$, and the undetermined function $h(z)$ is as stated in

$$
h(z)=\left\{\begin{array}{l}
0, z \in \Gamma, \\
h_{j}, z \in \Gamma_{j}, j=1, \cdots, N-K, \\
0, z \in \Gamma_{j}, j=N-K+1, \cdots, N+1 \\
h_{j}, z \in \Gamma_{j}, j=1, \cdots, N, \\
h_{0}+\operatorname{Re} \sum_{m=1}^{-K-1}\left(h_{m}^{+}+i h_{m}^{-}\right) z^{m}, z \in \Gamma_{0}
\end{array}\right\} \quad K<K<N,
$$

in which $h_{j}(j=0,1, \cdots, N), \quad h_{m}^{ \pm}(m=1, \cdots,-K-1, K<0)$ are unknown real constants to be determined appropriately. In addition, for $K \geq 0$ the solution $w(z)$ is assumed to satisfy the point conditions

$$
\begin{aligned}
& \operatorname{Im}\left[\overline{\lambda\left(a_{j}\right)} w\left(a_{j}\right)\right]=b_{j}, \\
& j \in J= \begin{cases}1, \cdots, 2 K-N+1, & K \geq N, \\
N-K+1, \cdots, N+1, & 0 \leq K<N,\end{cases}
\end{aligned}
$$

where

$$
\begin{aligned}
& a_{j} \in \Gamma_{j}(j=1, \cdots, N), \\
& a_{j} \in \Gamma_{0}(j=N+1, \cdots, 2 K-N+1, K \geq N)
\end{aligned}
$$

are distinct points, and $b_{j}(j \in J+\{0\})$ are all real constants satisfying the conditions

$$
\left|b_{j}\right| \leq k_{3}, j \in J+\{0\},
$$

for a non-negative constant $k_{3}$.

\section{Estimates of Solutions for the Poincaré Boundary Value Problem}

First of all, we give a prior estimate of solutions of Problem $Q$ for (1.6).

Theorem 2.1. Suppose that Condition $C$ holds and $\varepsilon=$ 0 in (1.6) and (1.7). Then any solution $[w(z), u(z)]$ of Problem $Q$ for (1.6) satisfies the estimates

$$
\begin{gathered}
C_{\beta}[w(z), \bar{D}]+C_{\beta}[u(z), \bar{D}] \leq M_{1} k^{*}, \\
L_{p_{0}, 2}\left[\left|w_{\bar{z}}\right|+\left|w_{z}\right|, \bar{D}\right] \leq M_{2} k^{*},
\end{gathered}
$$

in which

$$
\begin{gathered}
\beta=\min \left(\alpha, 1-2 / p_{0}\right), \\
M_{j}=M_{j}\left(q_{0}, p_{0}, k_{0}, \alpha, K, D\right), j=1,2, \\
k^{*}=k_{1}+k_{2}+k_{3}+k_{0}\left\{[C(w, \bar{D})]^{\sigma}+[C(u, \bar{D})]^{\tau}\right\} .
\end{gathered}
$$

Proof. Noting that the solution $[w(z), u(z)]$ of Problem $Q$ satisfies the equation and boundary conditions

$$
\begin{gathered}
w_{\bar{z}}-\operatorname{Re}\left[Q w_{z}+A_{1} w\right]=A_{3}+G(z, u, w) \text { in } D, \\
\operatorname{Re}[\overline{\lambda(z)} w]=c_{2}(z)+h(z) \text { on } \Gamma, \\
\operatorname{Im}\left[\overline{\lambda\left(a_{j}\right)} w\left(a_{j}\right)\right]=b_{j}, j \in J, u(1)=b_{0},
\end{gathered}
$$

according to the method in the proof of Theorem 4.3, Chapter II, [2] or Theorem 2.2.1, [5], we can derive that the solution $w(z)$ satisfies the estimates

$$
\begin{gathered}
C_{\beta}[w(z), \bar{D}] \leq M_{3} k_{*}, \\
L_{p_{0}, 2}\left[\left|w_{\bar{z}}\right|+\left|w_{z}\right|, \bar{D}\right] \leq M_{4} k_{*},
\end{gathered}
$$


where

$$
M_{j}=M_{j}\left(q_{0}, p_{0}, k_{0}, \alpha, K, D\right), j=3,4
$$

and

$$
k_{*}=k_{1}+k_{2}+k_{3}+L_{p}[G, \bar{D}] .
$$

From (1.8), it follows that

$$
\begin{gathered}
C_{\beta}[u(z), \bar{D}] \leq M_{5} C_{\beta}[w(z), \bar{D}]+k_{3}, \\
L_{p_{0}, 2}\left[\left|u_{\bar{z}}\right|+\left|u_{z}\right|, \bar{D}\right] \leq M_{5} C_{\beta}[w(z), \bar{D}]+k_{3},
\end{gathered}
$$

in which $M_{5}=M_{5}\left(p_{0}, D\right)$ is a non-negative constant. Moreover, it is easy to see that

$$
\begin{aligned}
L_{p, 2}[G, \bar{D}] \leq & L_{p, 2}\left[B_{1}, \bar{D}\right][C(w, \bar{D})]^{\sigma} \\
& +L_{p, 2}\left[B_{2}, \bar{D}\right][C(u, \bar{D})]^{\tau} \\
\leq & k_{0}\left\{[C(w, \bar{D})]^{\sigma}+[C(u, \bar{D})]^{\tau}\right\} .
\end{aligned}
$$

Combining (2.6)-(2.10), the estimates (2.1) and (2.2) are obtained.

Theorem 2.2. Let the Equation (1.6) satisfy Condition $C$ and $\varepsilon$ in (1.6)-(1.7) be small enough. Then any solu- tion $[w(z), u(z)]$ of Problem $Q$ for (1.6) satisfies the estimates

$$
C_{\beta}[w(z), \bar{D}]+C_{\beta}[u(z), \bar{D}] \leq M_{6} k^{*},
$$

$$
L_{p_{0}, 2}\left[\left|w_{\bar{z}}\right|+\left|w_{z}\right|, \bar{D}\right]+L_{p_{0}, 2}\left[u_{z}, \bar{D}\right] \leq M_{7} k^{*},
$$

here $\beta, p_{0}, k^{*}$ are as stated in Theorem 2.1,

$$
M_{j}=M_{j}\left(q_{0}, p_{0}, k_{0}, \alpha, K, D\right), j=6,7 .
$$

Proof. It is easy to see that $[w(z), u(z)]$ satisfies the equation and boundary conditions

$$
\begin{gathered}
w_{\bar{z}}-\operatorname{Re}\left[Q w_{z}\right]+A_{1} w=\varepsilon A_{2} u+A_{3}+G, z \in D, \\
\operatorname{Re}[\overline{\lambda(z)} w(z)]=-\varepsilon c_{1} u+c_{2}(z)+h(z), z \in \Gamma, \\
\operatorname{Im}\left[\overline{\lambda\left(a_{j}\right)} w\left(a_{j}\right)\right]=b_{j}, j \in J, u(1)=b_{0} .
\end{gathered}
$$

Moreover from (2.6) and (2.7), we have

$$
\left\{\begin{array}{l}
C_{\beta}[w(z), \bar{D}] \leq M_{3}\left\{k^{*}+\varepsilon k_{0} C_{\beta}[u, \bar{D}]\right\}, \\
L_{p_{0}, 2}\left[\left|w_{\bar{z}}\right|+\left|w_{z}\right|, \bar{D}\right] \leq M_{4}\left\{k^{*}+\varepsilon k_{0} C_{\beta}[u, \bar{D}]\right\},
\end{array}\right.
$$

and from (2.8)-(2.10), it follows that

$$
\left\{\begin{array}{l}
C_{\beta}[w(z), \bar{D}] \leq M_{3}\left\{k^{*}+\varepsilon k_{0}\left[M_{5} C_{\beta}[w(z), \bar{D}]+k_{3}\right]\right\}, \\
L_{p_{0}, 2}\left[\left|w_{\bar{z}}\right|+\left|w_{z}\right|, \bar{D}\right] \leq M_{4}\left\{k^{*}+\varepsilon k_{0}\left[M_{5} C_{\beta}[w(z), \bar{D}]+k_{3}\right]\right\} .
\end{array}\right.
$$

If the positive constant $\varepsilon$ is small enough such that $1-\varepsilon k_{0} M_{3} M_{5} \geq 1 / 2$, then the first inequality in (2.17) implies that

$$
\begin{aligned}
& \qquad \begin{array}{ll}
C_{\beta}[w(z), \bar{D}] \leq & \frac{\left(1+\varepsilon k_{0}\right) M_{3}}{1-\varepsilon k_{0} M_{3} M_{5}} k^{*} \\
\leq 2\left(1+\varepsilon k_{0}\right) M_{3} k^{*}=M_{8} k^{*} . & \begin{array}{l}
\text { (2.18) } \\
\text { which is the estived from (2. } \\
\text { i.e. }
\end{array} \\
\qquad L_{p_{0}, 2}\left[\left|w_{\bar{z}}\right|+\left|w_{z}\right|, \bar{D}\right]+L_{p_{0}, 2}\left[u_{z}, \bar{D}\right] \\
\leq M_{4}\left\{k^{*}+\varepsilon k_{0}\left[M_{5} C_{\beta}[w(z), \bar{D}]+k_{3}\right]\right\}+M_{5} C_{\beta}[w \\
\leq\left[1+M_{4}\left(1+\varepsilon k_{0}\right)+M_{5} M_{8}\left(1+\varepsilon k_{0} M_{4}\right)\right] k^{*}=M_{7} k^{*} .
\end{array} \\
& \text { 3. Solvability Results of the Poincaré } \\
& \text { Boundary Value Problem }
\end{aligned}
$$

\section{Solvability Results of the Poincaré Boundary Value Problem}$$
\leq M_{4}\left\{k^{*}+\varepsilon k_{0}\left[M_{5} C_{\beta}[w(z), \bar{D}]+k_{3}\right]\right\}+M_{5} C_{\beta}[w(z), \bar{D}]+k_{3}
$$

We first prove a lemma.

Lemma 3.1. If $G(z, u, w)$ satisfies the condition stat-
Combining (2.8) and (2.18), we obtain

$$
\begin{aligned}
& C_{\beta}[w(z), \bar{D}]+C_{\beta}[u(z), \bar{D}] \\
& \leq\left[1+\left(1+M_{5}\right) M_{8}\right] k^{*}=M_{6} k^{*},
\end{aligned}
$$

which is the estimate (2.11). As for (2.12), it is easily derived from (2.9) and the second inequality in (2.17), i.e.

$$
L_{p, 2}[G(z, u(z), w(z)), \bar{D}] \leq L_{p, 2}\left[B_{1}, \bar{D}\right][C(w, \bar{D})]^{\sigma}+L_{p, 2}\left[B_{2}, \bar{D}\right][C(u, \bar{D})]^{\tau},
$$


where $p=p_{0}>2$.

Proof. In order to prove that the mapping $G$ :

$$
C(\bar{D}) \times C(\bar{D}) \rightarrow L_{p, 2}(\bar{D})
$$

Defined by $G=G[z, u(z), w(z)]$ is continuous, we choose any sequence of functions $\left[w_{n}(z), u_{n}(z)\right]$

$$
\left(w_{n}(z), u_{n}(z) \in C(\bar{D}), n=0,1,2, \cdots\right)
$$

such that

$$
C\left[w_{n}-w_{0}, \bar{D}\right]+C\left[u_{n}-u_{0}, \bar{D}\right] \rightarrow 0
$$

as $n \rightarrow \infty$. Similarly to Lemma 2.2.1, [5], we can prove that

$$
C_{n}=G\left(z, u_{n}, w_{n}\right)-G\left(z, u_{0}, w_{0}\right)
$$

possesses the property

$$
L_{p, 2}\left[C_{n}, \bar{D}\right] \rightarrow 0 \text { as } n \rightarrow \infty \text {. }
$$

$$
\left\{\begin{array}{l}
F\left(z, u_{1}, w_{1}, V\right)-F\left(z, u_{2}, w_{2}, V\right)=\operatorname{Re}\left[\tilde{A}_{1}\left(w_{1}-w_{2}\right)\right]+\varepsilon \tilde{A}_{2}\left(u_{1}-u_{2}\right), \\
G\left(z, u_{1}, w_{1}\right)-G\left(z, u_{2}, w_{2}\right)=\operatorname{Re}\left[\tilde{B}_{1}\left(w_{1}-w_{2}\right)\right]+\varepsilon \tilde{B}_{2}\left(u_{1}-u_{2}\right)
\end{array}\right.
$$

where

$$
L_{p, 2}\left[\tilde{A}_{j}, \bar{D}\right], L_{p, 2}\left[\tilde{B}_{j}, \bar{D}\right] \leq k_{0}<\infty, j=1,2,
$$

$\varepsilon$ is a sufficiently small positive constant, then the
And the inequality (3.1) is obviously true.

Theorem 3.2. Let the complex Equation (1.1) satisfy Condition $C$, and the positive constant $\varepsilon$ in (1.6) and (1.7) is small enough.

1) When $0<\sigma, \tau<1$, Problem $Q$ for (1.6) has a solution $[w(z), u(z)]$, where $w(z), u(z) \in W_{p_{0}, 2}^{1}(D)$, $p_{0}\left(2<p_{0} \leq p\right)$ is a constant as stated before.

2) When $\min (\sigma, \tau)>1$, Problem $Q$ for (1.6) has a solution $[w(z), u(z)]$, where $w(z) \in W_{p_{0}, 2}^{1}(D)$, provided that

$$
M_{9}=L_{p, 2}\left[A_{3}, \bar{D}\right]+C_{\alpha}\left[c_{2}, \Gamma\right]+\sum_{j \in J+\{0\}}\left|b_{j}\right|
$$

is sufficiently small.

3) If $F\left(z, u, w, w_{z}\right), G(z, u, w)$ satisfy the conditions, i.e. Condition $C$ and for any functions $w_{j}(z)$,

$u_{j}(z) \in C(\bar{D})(j=1,2)$ and $V(z) \in L_{p_{0}, 2}(\bar{D})$, there are

$$
\left(M_{6}+M_{7}\right)\left\{L_{p, 2}\left[A_{3}, \bar{D}\right]+L_{p, 2}\left[B_{1}, \bar{D}\right] t^{\sigma}+L_{p, 2}\left[B_{2}, \bar{D}\right] t^{\tau}+L_{\alpha}\left[a_{2}, \Gamma\right]+\sum_{j \in J+\{0\}}\left|b_{j}\right|\right\}=t,
$$

where $M_{6}, M_{7}$ are constants as stated in (2.11) and (2.12). Because $0<\sigma, \tau<1$, the Equation (3.5) has a unique solution $t=M_{10}>0$. Now we introduce a bounded, closed and convex subset $B^{*}$ of the Banach space $C(\bar{D}) \times$ $C(\bar{D})$, whose elements are of the form $[w(z), u(z)]$ satisfying the condition

$$
w(z), u(z) \in C(\bar{D}), C[w(z), \bar{D}]+C[u(z), \bar{D}] \leq M_{10} .
$$

We choose a pair of functions $[\tilde{w}(z), \tilde{u}(z)] \in B^{*}$ and substitute it into the appropriate positions of

$F\left(z, u, w, w_{z}\right), G(z, u, w)$ in (1.6) and the boundary condition (1.7), and obtain

$$
\begin{gathered}
w_{\bar{z}}=\tilde{F}\left(z, u, w, \tilde{u}, \tilde{w}, w_{z}\right)+G(z, \tilde{u}, \tilde{w}), \\
\operatorname{Re}[\overline{\lambda(z)} w(z)]=-\varepsilon c_{1}(z) \tilde{u}+c_{2}(z)+h(z), z \in \Gamma,
\end{gathered}
$$

above solution of Problem $Q$ is unique.

Proof. 1) In this case, the algebraic equation for $t$ is as follows where

$$
\begin{aligned}
\tilde{F}\left(z, u, w, \tilde{u}, \tilde{w}, w_{z}\right)= & \operatorname{Re}\left[Q\left(z, \tilde{u}, \tilde{w}, w_{z}\right) w_{z}+A_{1}(z, \tilde{u}, \tilde{w}) w\right] \\
& +\varepsilon A_{2}(z, \tilde{u}, \tilde{w}) u+A_{3}(z, \tilde{u}, \tilde{w}) .
\end{aligned}
$$

In accordance with the method in the proof of Theorem 1.2.5, [5], we can prove that the boundary value problem (3.7), (3.8) and (1.6) has a unique solution $[w(z), u(z)]$. Denote by $[w, u]=T[\tilde{w}(z), \tilde{u}(z)]$ the mapping from $[\tilde{w}(z), \tilde{u}(z)]$ to $[w(z), u(z)]$. Noting that

$$
L_{p, 2}\left[\varepsilon A_{2} u, \bar{D}\right] \leq \varepsilon M_{10} k_{0}, C_{\alpha}\left[-\varepsilon c_{1} u, \Gamma\right] \leq \varepsilon M_{10} k_{0} .
$$

provided that the positive number $\varepsilon$ is sufficiently small, and noting that the coefficients of complex Equation (3.7) satisfy the same conditions as in Condition $C$, from Theorem 2.2, we can obtain

$$
\begin{aligned}
& C[w(z), \bar{D}]+L_{p_{0}, 2}\left[\left|w_{\bar{z}}\right|+\left|w_{z}\right|, \bar{D}\right]+C[u(z), \bar{D}]+L_{p_{0}, 2}\left[u_{z}, \bar{D}\right] \\
& \leq\left(M_{6}+M_{7}\right)\left\{L_{p, 2}\left[A_{3}, \bar{D}\right]+C_{\alpha}\left[c_{2}, \Gamma\right]+\sum_{j \in J+\{0\}}\left|b_{j}\right|+L_{p, 2}[G, \bar{D}]\right\} \\
& \leq\left(M_{6}+M_{7}\right)\left\{M_{9}+L_{p, 2}\left[B_{1}, \bar{D}\right] C[\tilde{w}, \bar{D}]^{\sigma}+L_{p, 2}\left[B_{2}, \bar{D}\right] C[\tilde{u}, \bar{D}]^{\tau}\right\} \\
& \leq\left(M_{6}+M_{7}\right)\left\{M_{9}+L_{p, 2}\left[B_{1}, \bar{D}\right] M_{10}^{\sigma}+L_{p, 2}\left[B_{2}, \bar{D}\right] M_{10}^{\tau}\right\}=M_{10} .
\end{aligned}
$$


This shows that $T$ maps $B^{*}$ onto a compact subset in $B^{*}$. Next, we verify that $T$ in $B^{*}$ is a continuous operator. In fact, we arbitrarily select a sequence $\left\{\tilde{w}_{n}(z), \tilde{u}_{n}(z)\right\}$ in $B^{*}$, such that

$$
C\left(\tilde{w}_{n}-\tilde{w}_{0}, \bar{D}\right)+C\left(\tilde{u}_{n}-\tilde{u}_{0}, \bar{D}\right) \rightarrow 0 \text { as } n \rightarrow \infty .
$$

By Lemma 3.1, we can see that

$$
\begin{aligned}
& L_{p, 2}\left[A_{j}\left(z, \tilde{u}_{n}, \tilde{w}_{n}\right)-A_{j}\left(z, \tilde{u}_{0}, \tilde{w}_{0}\right), \bar{D}\right] \rightarrow 0 \\
& (j=1,2,3) \text { as } n \rightarrow \infty .
\end{aligned}
$$

Moreover, from

$$
\begin{aligned}
& {\left[w_{n}, u_{n}\right]=T\left[\tilde{w}_{n}, \tilde{u}_{n}\right],} \\
& {\left[w_{0}, u_{0}\right]=T\left[\tilde{w}_{0}, \tilde{u}_{0}\right],}
\end{aligned}
$$

it is clear that $\left[w_{n}-w_{0}, u_{n}-u_{0}\right]$ is a solution of Problem $Q$ for the following equation

$$
\begin{aligned}
&\left(w_{n}-w_{0}\right)_{\bar{z}}= \tilde{F}\left(z, u_{n}, w_{n}, \tilde{u}_{n}, \tilde{w}_{n}, w_{n z}\right) \\
&-\tilde{F}\left(z, u_{0}, w_{0}, \tilde{u}_{0}, \tilde{w}_{0}, w_{0 z}\right) \\
&+G\left(z, \tilde{u}_{n}, \tilde{w}_{n}\right)-G\left(z, \tilde{u}_{0}, \tilde{w}_{0}\right) \text { in } D \\
& \operatorname{Re}\left[\overline{\lambda(z)}\left(w_{n}-w_{0}\right)\right] \\
&=-\varepsilon c_{1}(z)\left(\tilde{u}_{n}-\tilde{u}_{0}\right)+h(z) \text { on } \Gamma \\
& \operatorname{Im}\left[\overline{\lambda\left(a_{j}\right)}\left(w_{n}\left(a_{j}\right)-w_{0}\left(a_{j}\right)\right)\right]=0 \\
& j \in J, u_{n}(1)-u_{0}(1)=0 .
\end{aligned}
$$

In accordance with the method in proof of Theorem 2.2, we can obtain the estimate

$$
\begin{aligned}
& C\left[w_{n}-w_{0}, \bar{D}\right]+L_{p_{0}, 2}\left[\left|\left(w_{n}-w_{0}\right)_{\bar{z}}\right|+\left|\left(w_{n}-w_{0}\right)_{z}\right|, \bar{D}\right] \\
& +C\left[u_{n}-u_{0}, \bar{D}\right]+L_{p_{0}, 2}\left[\left(u_{n}-u_{0}\right)_{z}, \bar{D}\right] \\
& \leq M_{11}\left\{\varepsilon L_{p, 2}\left[A_{2}\left(z, \tilde{u}_{n}, \tilde{w}_{n}\right) \tilde{u}_{n}-A_{2}\left(z, \tilde{u}_{0}, \tilde{w}_{0}\right) \tilde{u}_{0}, \bar{D}\right]\right. \\
& +L_{p, 2}\left[A_{3}\left(z, \tilde{u}_{n}, \tilde{w}_{n}\right)-A_{3}\left(z, \tilde{u}_{0}, \tilde{w}_{0}\right), \bar{D}\right] \\
& +L_{p, 2}\left[G\left(z, \tilde{u}_{n}, \tilde{w}_{n}\right)-G\left(z, \tilde{u}_{0}, \tilde{w}_{0}\right), \bar{D}\right] \\
& \left.+\varepsilon C_{\alpha}\left[c_{1}(z)\left(\tilde{u}_{n}-\tilde{u}_{0}\right), \Gamma\right]\right\},
\end{aligned}
$$

in which $M_{11}=M_{11}\left(q_{0}, p_{0}, k_{0}, \alpha, K, D\right)$. From (3.10), (3.11) and the above estimate, we obtain

$$
C\left[w_{n}-w_{0}, \bar{D}\right]+C\left[u_{n}-u_{0}, \bar{D}\right] \rightarrow 0 \text { as } n \rightarrow \infty .
$$

On the basis of the Schauder fixed-point theorem, there exists a function $[w(z), u(z)](w(z), u(z) \in C(\bar{D}))$ such that $[w(z), u(z)]=T[w(z), u(z)]$, and from Theorem 2.2 , it is easy to see that $w(z), u(z) \in W_{p_{0}, 2}^{1}(D)$, and $[w(z), u(z)]$ is a solution of Problem $Q$ for the Equation (1.6) and the relation (1.8) with the condition $0<\sigma$, $\tau<1$.
In addition, if $G(z, u, w)=\operatorname{Re} B_{1} w+B_{2}|u|^{\tau}$ in $D$, where $0<\tau<1, L_{p, 2}\left[B_{j}, \bar{D}\right] \leq k_{0}<\infty, j=1,2$, then the above solvability result still hold by using the above similar method.

2) Secondly, we discuss the case: $\min (\sigma, \tau)>1$. In this case, (3.5) has the solution $t=M_{10}$ provided that $M_{9}$ in (3.3) is small enough. Now we consider a closed and convex subset $B_{*}$ in the Banach space $C(\bar{D}) \times C(\bar{D})$, i.e.

$$
B_{*}=\left\{w(z), u(z) \in C(\bar{D}), C[w, \bar{D}]+C[u, \bar{D}] \leq M_{10}\right\} .
$$

Applying a method similar as before, we can verify that there exists a solution

$$
[w(z), u(z)] \in W_{p_{0}, 2}^{1}(D) \times W_{p_{0}, 2}^{1}(D)
$$

of Problem $Q$ for (1.6) with the condition $\min (\sigma, \tau)>1$.

Moreover, if $G(z, u, w)=\operatorname{Re} B_{1} w+B_{2}|u|^{\tau}$ in $D$, where $1<\tau<\infty, L_{p, 2}\left[B_{j}, \bar{D}\right] \leq k_{0}<\infty, j=1,2$. Under the same condition, we can derive the above solvability result by the similar method.

3) When $G(z, u, w)$ satisfies the condition (3.4), we can verify the uniqueness of solutions in this theorem. In fact, if $\left[w_{1}(z), u_{1}(z)\right],\left[w_{2}(z), u_{2}(z)\right]$ are two solutions of Problem $Q$ for the Equation (1.6), then

$$
[w(z), u(z)]=\left[w_{1}(z)-w_{2}(z), u_{1}(z)-u_{2}(z)\right]
$$

satisfies the equation and boundary conditions

$$
\begin{gathered}
w_{\bar{z}}-\operatorname{Re}\left[\tilde{Q}_{w_{z}}+\left(\tilde{A}_{1}+\tilde{B}_{1}\right) w\right]=\varepsilon\left(\tilde{A}_{2}+\tilde{B}_{2}\right) u, z \in D, \\
\operatorname{Re}[\overline{\lambda(z)} w(z)]=-\varepsilon c_{1} u+h(z), z \in \Gamma, \\
\operatorname{Im}\left[\overline{\lambda\left(a_{j}\right)} w\left(a_{j}\right)\right]=0, j \in J .
\end{gathered}
$$

in which $|\tilde{Q}| \leq q_{0}<1$. Similarly to Theorem 2.2 , we can derive the following estimates of the solution $[w(z), u(z)]$ for complex Equation (3.17):

$$
\begin{gathered}
C_{\beta}[w(z), \bar{D}]+C_{\beta}[u(z), \bar{D}] \leq M_{12} k^{*}, \\
L_{p_{0}, 2}\left[\left|w_{\bar{z}}\right|+\left|w_{z}\right|, \bar{D}\right] \leq M_{13} k^{*},
\end{gathered}
$$

where

$$
\begin{gathered}
\beta=\min \left(\alpha, 1-2 / p_{0}\right), \\
M_{j}\left(=M_{j}\left(q_{0}, p_{0}, k_{0}, \alpha, K, D\right), j=12,13\right)
\end{gathered}
$$

are two non-negative constants, $k^{*}=2 \varepsilon k_{0} C(u, \bar{D})$. Moreover the estimate

$$
C_{\beta}[w(z), \bar{D}]+\left(1-2 \varepsilon k_{0} M_{13}\right) C_{\beta}[u(z), \bar{D}] \leq 0
$$

can be derived. Provided that the positive constant $\varepsilon$ is 
small enough such that $\left(1-2 \varepsilon k_{0} M_{13}\right)>0$, from (3.22) it follows $u(z)=u_{1}(z)-u_{2}(z)=0$, i.e. $u_{1}(z)=u_{2}(z)$ in $D$. This completes the proof of the theorem.

From the above theorem, the next result can be derived.

Theorem 3.3. Under the same conditions as in Theorem 3.2, the following statements hold.

1) When the index $K>N$, Problem $P$ for (1.1) has $N$ solvability conditions, and the solution of Problem $P$ depends on $2 K-N+2$ arbitrary real constants.

2) When $0 \leq K<N$, Problem $P$ for (1.1) is solvable, if $2 N-K$ solvability conditions are satisfied, and the solution of Problem $P$ depends on $K+2$ arbitrary real constants.

3) When $K<0$, Problem $P$ for (1.1) is solvable under $2 N-2 K-1$ conditions, and the solution of Problem $P$ depends on 1 arbitrary real constant.

Moreover, we can write down the solvability conditions of Problem $P$ for all other cases.

Proof. Let the solution $[w(z), u(z)]$ of Problem $Q$ for (1.6) be substituted into the boundary condition (1.7) and the relation (1.8). If the function $h(z)=0, z \in \Gamma$, i.e.

$$
\begin{cases}h_{j}=0, j=1, \cdots, N-K, & \text { if } 0 \leq K<N \\ h_{j}=0, j=0,1, \cdots, N, h_{m}^{ \pm}=0, m=1, \cdots,-K-1, & \text { if } K<0\end{cases}
$$

and $d_{j}=0, j=1, \cdots, N$, then we have $w(z)=u_{z}$ in $D$ and the function $w(z)$ is just a solution of Problem $P$ for (1.1). Hence the total number of above equalities is just the number of solvability conditions as stated in this theorem. Also note that the real constants $b_{0}$ in (1.8) and $b_{j}(j \in J)$ in (1.9) are arbitrarily chosen. This shows that the general solution of Problem $P$ for (1.1) includes the number of arbitrary real constants as stated in the theorem.

\section{REFERENCES}

[1] I. N. Vekua, "Generalized Analytic Functions," Pergamon, Oxford, 1962.

[2] G. C. Wen and H. Begehr, "Boundary Value Problems for Elliptic Equations and Systems," Longman Scientific and Technical Company, Harlow, 1990.
[3] A. V. Bitsadze, "Some Classes of Partial Differential Equations," Gordon and Breach, New York, 1988.

[4] G. C. Wen, "Conformal Mappings and Boundary Value Problems," Translations of Mathematics Monographs 106, American Mathematical Society, Providence, 1992.

[5] G. C. Wen, D. C. Chen and Z. L. Xu, "Nonlinear Complex Analysis and Its Applications," Mathematics Monograph Series 12, Science Press, Beijing, 2008.

[6] G. C. Wen, "Approximate Methods and Numerical Analysis for Elliptic Complex Equations," Gordon and Breach, Amsterdam, 1999.

[7] G. C. Wen, "Linear and Quasilinear Complex Equations of Hyperbolic and Mixed Type," Taylor \& Francis, London, 2002. doi: 10.4324/9780203166581

[8] G. C. Wen, "Recent Progress in Theory and Applications of Modern Complex Analysis," Science Press, Beijing, 2010. 